Article

\title{
Augmentative and Alternative Communication (AAC) Advances: A Review of Configurations for Speech Disabled Individuals
}

\author{
Yasmin Elsahar, Sijung Hu*, Kaddour Bouazza-Marouf, David Kerr, Annysa Mansor \\ Wolfson School of Mechanical, Electrical, and Manufacturing Engineering, Loughborough University, \\ Loughborough, UK, LE11 3TU \\ * Correspondence: S.Hu@lboro.ac.uk; Tel.: +44-150-922-7058
}

\begin{abstract}
High-tech augmentative and alternative communication (AAC) methods are on a constant rise; however, the interaction between the user and the assistive technology is still challenged for an optimal user experience centered around the desired activity. This review presents a range of signal sensing and acquisition methods utilized in conjunction with the existing high-tech AAC platforms for speech disabled individuals, including imaging methods, touch-enabled systems, mechanical and electro-mechanical access, breath-activated methods, and brain computer interfaces (BCI). The listed AAC sensing modalities are compared in terms of ease of access, affordability, complexity, portability, and typical conversational speeds. A revelation of the associated AAC signal processing, encoding, and retrieval highlights the roles of machine learning (ML) and deep learning (DL) in the development of intelligent AAC solutions. The demands and the affordability of most systems were found to hinder the scale of usage of high-tech AAC. Further research is indeed needed for the development of intelligent AAC applications reducing the associated costs and enhancing the portability of the solutions for a real user's environment. The consolidation of natural language processing with current solutions also needs to be further explored for the amelioration of the conversational speeds. The recommendations for prospective advances in coming high-tech AAC are addressed in terms of developments to support mobile health communicative applications.
\end{abstract}

Keywords: augmentative and alternative communication; assistive technologies; sensing modalities; signal processing; voice communication; machine learning; mobile health; speech disability

\section{Introduction}

Recent studies show that up to $1 \%$ of the world population suffers a degree of speech, language or communication need (SLCN) [1,2]. The loss of speech capabilities associated with extreme forms of paralysis and further medical complications has long been regarded as a barrier between the sufferers and the outside world. Augmentative and alternative communication (AAC) incorporates a wide range of processes that augment, complement, or replace speech of individuals with complex communication needs [3,4]. In the broad context of speech and language, speech is often associated with the motor movements responsible for the production of spoken words, whereas language is associated with the cognitive processing skills of communication.

AAC solutions are classified into three categories: no-tech, low-tech, and high-tech AAC [4]. No-tech AAC is considered the oldest of the three AAC categories, given its reliance on the interpretation of facial expressions and voluntary motor movements, such as sign language, to deliver non-verbal messages [5]. Low-tech AAC utilizes basic tools, such as books and display boards with extended lexicons of images and phrases to aid the communication process [6]. High-tech AAC 
encompasses the use of electronic devices to achieve an AAC target. Devices falling under this category, such as smart devices and dedicated AAC devices, integrate hardware and software to support a user's communication needs. A common attribute of modern day AAC solutions tends to rely on the translation of a user's intended meanings into speech via speech generating devices (SGDs) [4]. AAC communication is also often classified as either un-aided or aided, given the dependence of the solution on the human body solely or the interaction with an external communicative aid for communication respectively [4].

The potential of AAC intervention has hence been substantial over the last 30 years, with the provision of innovative solutions to a wide range of speech-impaired users [7]. However, although high-tech AAC systems are rapidly evolving, several considerations are yet pertinent to the provision of effective solutions efficiently serving AAC users $[4,8]$. Low-tech AAC solutions are usually the first techniques tried by speech and language therapists, as the use of simplistic display boards and communication books is both cost-effective and easy to obtain. Moreover, the high costs and complicated training a user requires to operate most high-tech AAC devices could hinder the access to high-tech AAC, and thus the usability of speech generating devices. In turn, an optimized use of high-tech AAC should be researched to provide a faster means of communication, in comparison to low-tech, by prioritizing the communicative needs of the users over the needs of the system. Studies also show that after testing several AAC systems, the potential of AAC might be limited by complex operational difficulties given the number of users who are simultaneously physically impaired and speech-disabled [8]. Predominantly, AAC users still use combinations of unaided low-tech methods together with an aided high-tech device as suitable for the context of usage and the person they are conversing with [9].

Due to the complex composition of the human body, speech and communication impairments requiring an AAC intervention could result from diverse medical conditions $[10,11]$. These commonly include Autistic Spectrum Disorders (ASD), strokes, learning disabilities, Locked-in-Syndrome (LIS), Dementia, head and neck cancers, and brain injuries. This also expands to include patients with progressive diseases, such as Parkinson's Disease and Amyotrophic Lateral Sclerosis (ALS) [10]. Other AAC users include patients in transient post-operative states where interventions and treatments, such as ventilator support, may render them unable to speak normally, or at all. In turn, the users benefiting from AAC intervention could be classified into three major groups based on their individual conditions and the intended target use of the AAC communicative aid [12]. These three classes comprise alternative-language users, augmentative-language users, and temporary AAC users. Alternative-language users have a well-established cognitive understanding of language and speech, but have difficulties in conversing. On the other hand, augmentative-language users have difficulties both in understanding speech and in conversing. To be able to use an AAC device, augmentative-language users need assistance in the re-categorization of their surroundings into labels and symbols they comprehend to form a communication language. Temporary AAC users require AAC intervention only for a limited duration of time. This category primarily includes children with developmental conditions, and adults who require transient speech assistance following surgical intervention [12].

Given the complexity of the user base, and the wide need for AAC solutions to serve diverse groups of speech disabled individuals, current research efforts are being redirected towards the establishment of assistive systems that are suited to respond to their personal users' needs and capabilities. The aim of this study is to review the access and processing techniques pertaining to predominant high-tech AAC methods, including the input signal sources, and the developments of machine learning (ML) and deep learning (DL) associated with AAC solutions for the provision of a personalized user experience. 


\section{Human Interaction}

Several studies exist in the literature of modelling the user's interaction with assistive technologies (AT) [13]. A primary, well-established, AT framework is the Human Activity Assistive Technology (HAAT) model [4]. The HAAT model underpins a consolidated approach of the interactions between the activity, the human, the context, and the assistive technology. It links the process of selection of an assistive technology solution with the person carrying out an activity in a given context [14]. The four components constituting the HAAT model are shown in Figure 1. A particular attention is drawn to each component, detailing the importance of firstly considering the target activity (self-care, productivity, leisure), the human abilities of the person using the device (physical, cognitive, emotional, and expertise), the context the device is used in (physical, cultural, institutional), and in turn the consideration of the suitable AT device (interfaces, processor, output) [4]. The developers of the HAAT model emphasize on the concept of serving the needs of the users in order to optimize the usage of the technology, stating that the technology aspect should encompass the function it serves, the person who will be using the AT device, and the context of usage [4].

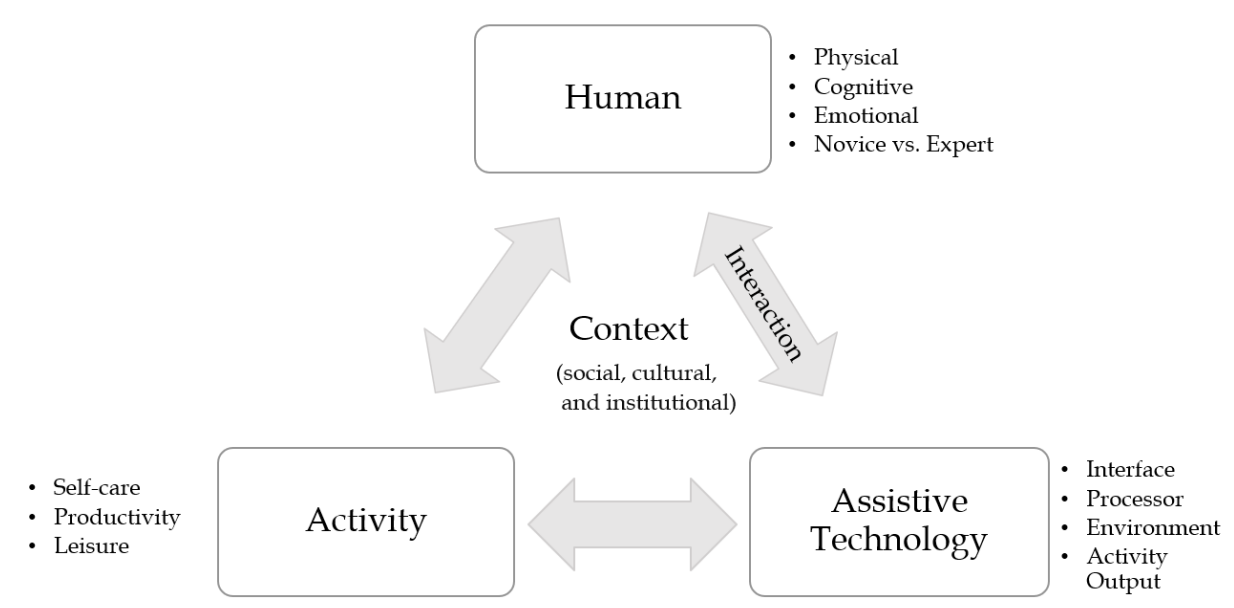

Figure 1. The four components of the Human Activity Assistive Technology (HAAT) model, from [4]. The interaction between the human and the assistive technology (AT) is emphasized to highlight the relationship between the needs of the AAC users and the elements of development of high-tech solutions discussed in this review.

In light of the HAAT model, AT could hence be used to aid the communication process of speech disabled individuals, given that the technology prioritizes the activities and abilities of the user. Basing high-tech AAC applications and platforms on the skills and communicative needs of the users, disabled persons could in turn be allowed to participate in a wider range of activities to communicate their individual needs [15]. From the societal perspective, smart devices have been promoting both the visibility and acceptance of AAC [16]. A number of factors also aids in increasing the access to high-tech AAC platforms, including the ease of operating and using the AAC device, its processing capabilities, the cost of the hardware, and the licensed software packages used to operate the devices [4]. Emphasis is also placed through several studies [4,17] on the importance of customizing AT solutions to address the needs of the users who might find difficulties in accessing the devices' interfaces. A survey study in [18] further highlighted the importance of the provision of technical support and the time taken by a device to communicate a message.

\section{Sensing Modalities and their Functionalities}

The integration of smart developments into daily life activities has widened the scope of dedicated and non-dedicated AAC applications [7,19]. A survey of high-tech AAC devices with regards to the signal acquisition, ML, and output generation is presented in this section. 


\subsection{AAC Signal Sources and Associated Processing}

AAC interfaces are activated through an array of methods for the detection of human signals generated via body movements, respiration, phonation, or brain activities [4]. The acquisition of AAC signals is accomplished through several modalities. Table 1 outlines the AAC signal sensing categories discussed in this review together with their relevant activation methods. The listed AAC access methods could be used in a stand-alone format or in combination with one another. For example, imaging methods may be combined with touch-activated methods or mechanical switches to provide the users with a multi-modal access using the same device. A commercial example is Tobii Dynavox PCEye Plus, which combines several functionalities including eye tracking and switch access to use a computer screen [20].

Table 1. Sensing modalities of AAC signals

\begin{tabular}{ll}
\hline Signal sensing category & Activation method \\
\hline Imaging methods & Eye gaze systems, head-pointing devices \\
Mechanical and Electromechanical methods & Mechanical keyboards, switch access \\
Touch-activated methods & Touchscreens, touch membrane keyboards \\
Breath-activated methods & Microphones, low-pressure sensors \\
Brain Computer Interface methods & Invasive and non-invasive \\
\hline
\end{tabular}

\subsubsection{Imaging Methods}

Imaging methods, such as eye gazing, eye tracking and head-pointing devices, have been widely reported in the literature [21-31]. Eye gaze technologies work using the principle of tracking the eye movements of a user for the determination of the eye gaze direction [24,27]. Several eye tracking methods are commonly used, including video-oculography [32], electro-oculography [33], contact lenses [34], and electromagnetic scleral coils [21,25,30,35,36]. Oculography is involved with the measurement and recording of a user's eye movements [35]. Video-oculography and electro-oculography use video-based tracking systems and skin surface elecrodes respectively to track the movements of the eye [25]. In the context of AAC, non-invasive eye tracking methods are better suited to address the daily needs of the users who lack motor abilities [27,29]. Practical methods involve the utilization of non-invasive cameras, an illumination source, image processing algorithms, and speech synthesizers to communicate a user's message [25,27]. Image data is obtained in video-oculography operated system using one or more cameras [23,27]. Typical video-oculography systems use glints produced on the surface of the eye through an illumination source, such as near-infrared (NIR) LEDs with typical wavelengths of $850 \pm 30 \mathrm{~nm}$, and in turn, gaze locations are estimated from the movement of the eye pupil in relation to the illuminated glint positions [34].

The components of a typical video-based tracking system are shown in Figure 2. Different approaches are present in the literature of calculating the accuracy of an eye tracking system, including the distance accuracy (in cm or in pixels) and the angular accuracy (in degrees) [22]. The pixel accuracy can be given by

$$
P_{\text {acc }}=\sqrt{\left(X_{\text {target }} P X\right)^{2}+\left(Y_{\text {target }} P Y\right)^{2}}
$$

where $X_{\text {target }}$ and $Y_{\text {target }}$ are the coordinates of the target points, and PX and PY are the gaze point coordinates given by

$$
P X=\text { mean }\left(\frac{P X_{\text {left }}+P X_{\text {right }}}{2}\right)
$$


and

$$
P Y=\text { mean }\left(\frac{P Y_{l e f t}+P Y_{\text {right }}}{2}\right)
$$

respectively, with the subscripts left and right referring to the coordinates of gaze points of the left and the right eye. The on-screen distance accuracy $(D A)$ is similarly given by

$$
D A=p_{\text {size }} \sqrt{\left(P X-\frac{x_{\text {pixels }}}{2}\right)^{2}+\left(y_{\text {pixels }}-P Y+\frac{o f f s e t}{\text { pixelsize }}\right)^{2}}
$$

where $p_{\text {size }}$ is calculated based on the resolution, height, and width of the screen, $x_{\text {pixels }}$ and $y_{\text {pixels }}$ are the pixel shifts in the directions of $\mathrm{x}$ and $\mathrm{y}$ respectively, and the offset is the distance between the eye tracking unit and the lower edge of the screen $[22,37]$. The angular accuracy $(A A)$ can be also computed via

$$
A A=\frac{p_{\text {size }} \times P_{a c c} \times \cos (\text { mean }(\theta))^{2}}{\text { meandist }}
$$

where the gaze angle $\theta$ is given by

$$
\theta=\tan ^{-1}\left(\frac{D A}{d i s t}\right)
$$

and dist and meandist are the distances from the eye to the screen and from the eye to the tracker respectively $[22,37]$.

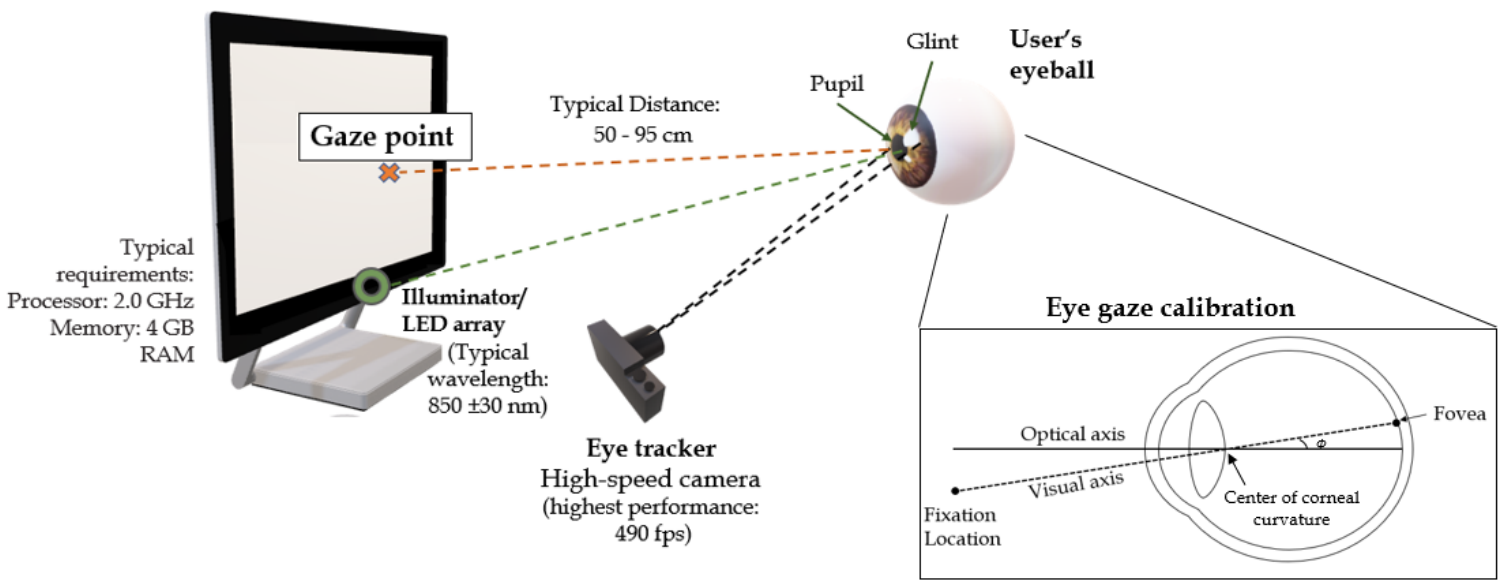

Figure 2. Components of a typical eye gaze system, adapted from [22,38]. The optical and the visual axes are used for the calibration process commonly required to set up the eye gaze system [22,39].

Fixations and saccades are commonly used to analyze eye movements [40]. Fixations are the pauses a user intently inputs by fixing his eye movements at the target gaze point, whereas saccades are the eye movements rapidly occurring following and in between the fixations. Metrics of eye gaze estimations include fixation durations, fixation rates, fixation sequences, saccadic amplitudes and velocities [22,40]. Although electro-oculography is a cost-effective eye tracking method, Infrared pupil corneal reflection (IR-PCR) video-based systems are most commonly used by speech and language practitioners due to their non-invasive nature [25,27]. A calibration operation is essential in video-based trackers to fine-tune the system with a user's eye movements [41]. As seen in Figure 2, a user's visual axis deviates from the optical axis upon the usage of a gaze system. Calibration is expressed as the process of finding the visual axis pertinent to each user by calculating the angle between the line joining the fovea (the highest point of sensitivity in the eye retina) with the center of corneal curvature, and the optical axis [22]. 
The estimation of the visual axis is usually not feasible, and as such, the calibration process enables the tracker to capture and learn the difference between the user's eye positions when gazing at a specific target in comparison to the actual coordinates of the gaze target. The user's head orientation should be also considered in IR-PCR systems, as the movements of the user's head can adversely impact the calculations of the glint vectors [22]. Studies are however addressing advances in eye tracking methods to overcome the related constraints, providing the forthcoming possibilities of free IR eye tracking and robust algorithms for head movements compensation [42].

\subsubsection{Mechanical and Electro-mechanical Methods}

Mechanical and electro-mechanical AAC devices have applications for both direct and indirect selection access methods. Direct selections offer the users sets of choices, and require a voluntary input selection of the intended messages from the user's side. This usually involves the coordination of voluntary controls using a body part, such as the hand or fingers, or a pointing device, to select a message [19]. Mechanically activated direct-selection methods include mechanical keyboards, which utilize the physical mechanical depression of the pressed keys to activate a user selection. Keyboard layouts maybe reconfigured for individuals who find the use of a standard keyboard difficult due to the required coordination between the two hands [4].

For individuals lacking voluntary controls, communication via direct selections is often cumbersome, and consequently, indirect selection methods are best-suited for this group of users [19]. Scanning methods are predominantly in use with indirect selections, involving a systematic representation of options appearing in timed intervals for the users to select from [19,43]. Mechanical scanning methods include single switches, arrays of switches, or other variations of methods activated via the application of a force [4]. Switches are generally considered a form of low-tech AAC due to their minimal hardware requirements; however, switching applications have recently expanded to allow users the access of several high-tech AAC platforms, including computers, tablets, or smart devices via scanning. Scanning techniques range across three levels, each suited to accommodate users with specific motor abilities: Automatic scanning is used to present items in adjustable time intervals, based on the user's skills, until a selection is made; step scanning allows the users to control the presentation of selections, in turn controlling the rate of advancement; and inverse scanning involves holding down a control interface and releasing it upon the desired selection [4]. Figure 3 shows a visual scanning interface together with typical activation switches.

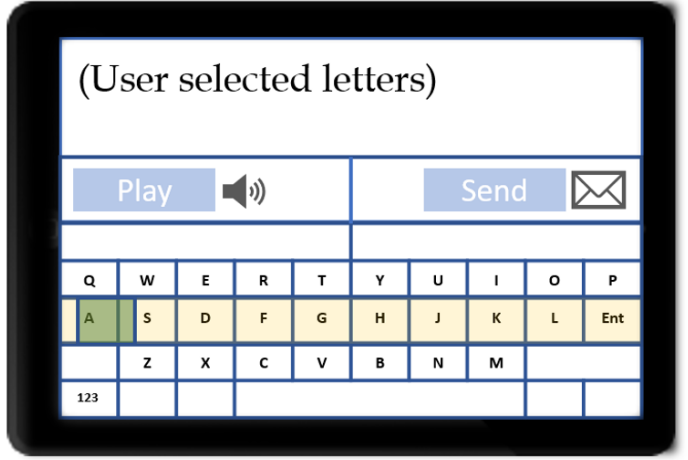

(a)

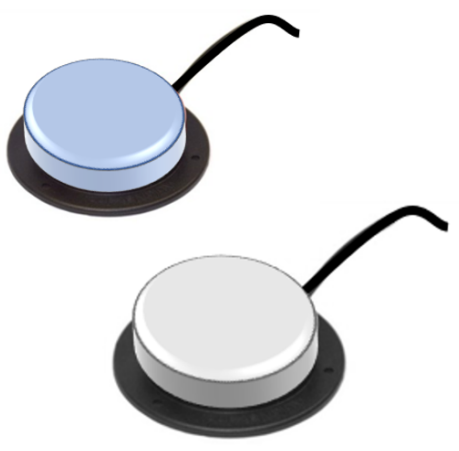

(b)

Figure 3. (a) A sample visual scanning interface activated via switch scanning. The yellow box moves vertically across the lines until a selection is made, followed by a gliding green box moving horizontally across the highlighted line until a letter is also selected. In (b), two scanning button switches are displayed.

In addition to letters, scanning interfaces expand to include a variety of access options, including icons, pre-stored messages, and auditory messages. Some operating systems also provide the option 
of device navigation via an external switch. The position and access methods of switches are user dependent. They can be adjusted to be in close proximity to the hands or the feet for the ease of activation. Mechanical switches can be also mounted on wheel chairs to allow access using head movements. Different variations of switches are available in terms of shapes and types to suit the user's requirements. In general, mechanical switch scanning requires minimal motor movements; however, the communicative rates could be slowed down by the delay required to make a selection.

\subsubsection{Touch-activated Systems}

With the escalation of the touchscreen developments, touch-activated AAC applications are commonly in use with AAC direct selection activation. Touchscreen technologies comprise various types, including resistive, capacitive, Surface Acoustic Wave, and optical/infrared touchscreens [44]. Resistive and capacitive touchscreens are predominantly used with smart devices [45]. Resistive touchscreens are dependent on the production of a force or pressure using the user's fingers, whereas capacitive touchscreens are activated using the electrical charge present on the user's finger [46]. Although resistive touchscreens are cost efficient, capacitive touchscreens are often known to present a better visual clarity, presenting an added benefit for AAC users suffering a degree of visual impairments. Touch membrane keyboards are also in use by AAC users. They are built using non-conductive spacers separating conductive flat surfaces; and acquire electronic signals through the pressure resulting from holding down a key, generating an input signal to the AAC device [19].

AAC users utilize touchscreens and touch activated systems to make selections via swiping and tapping; however, such actions could be restrictive for the users who are physically impaired [4]. Nonetheless, the accuracy can be augmented using pointers, as the icons presented on a touchscreen often have the advantage of being cognitively easy to select, and less demanding in comparison to the operation of a regular computer [4].

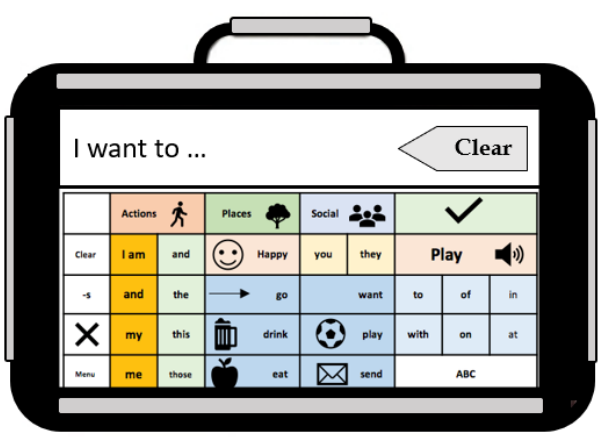

(a)

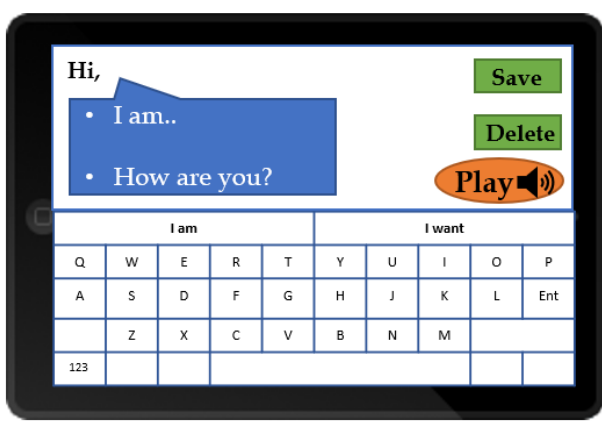

(b)

Figure 4. Examples of (a) a dedicated touch-based device and (b) a non-dedicated smart device running an AAC APP, usually with predictive language model and speech generation capabilities.

\subsubsection{Breath-activated Systems}

The wide availability of sensing modalities expands the scope of AAC control interfaces to include the detection of respiratory signals in addition to the regular voluntary body movements [4]. Voluntary body movements are commonly detected through the integration of sensors with imaging, and/or optical, mechanical, and electro-mechanical devices. Respiration signals are recorded via a wide range of modalities, including fibre optic sensors [47], pressure and thermal sensors [48], photoplethysmogram (PPG) measurements [49], electroencephalogram (EEG) signals [50], and the examination of airflow [50,51]. Discrete and continuous breathing signals can be used to encode messaged, as shown in figure 5. Discrete breath encoding involves the generation of soft and heavy breathing blows encoded as binary combinations of zeros and ones, or Morse codes to represent the user's intended messages or the International Morse code's letters respectively. On the other hand, continuous breath encoding uses the modulation of the speed, amplitude, and phase of breathing 
signals to create patterns representing the intended message. The modulation of the continuous breathing patterns encoded to represent user selected phrases, including the training and retrieval modes, is shown in Figure 6 for a mobile based APP.

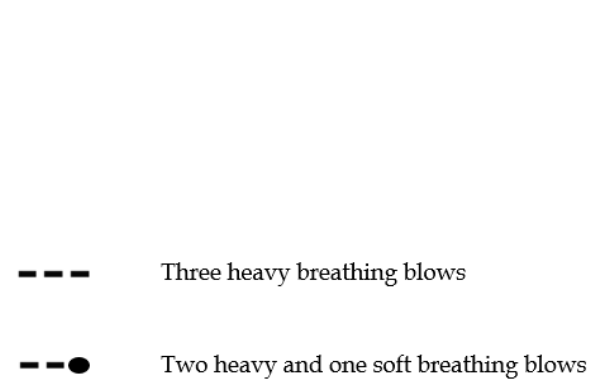

(a) Discrete breath-encoding

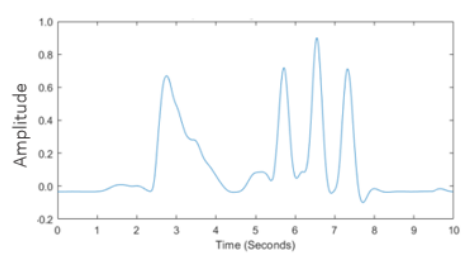

Modulated pattern, one slow and three fast breathing cycles

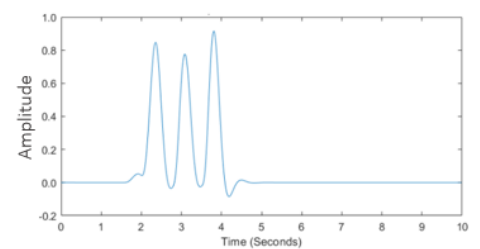

Modulated pattern, three fast breathing cycles

Figure 5. Examples of (a) discrete breath encoding, where soft and heavy breathing blows are recorded to encode combinations of zeros and ones, or Morse codes, representing the intended messages, and (b) continuous breath encoding, where the speed, amplitude, and phase of breathing are modulated to create patterns representing the intended message.
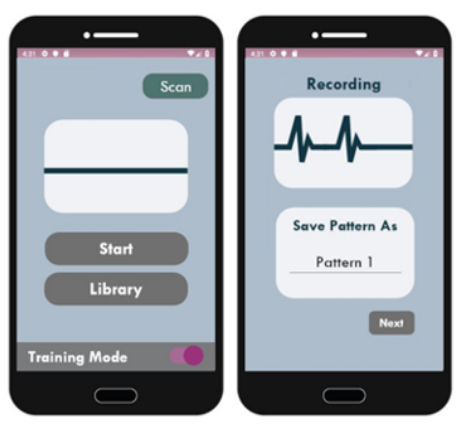

(a)
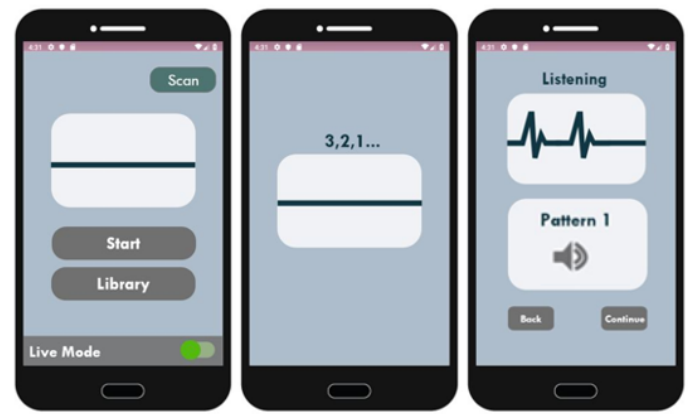

(b)

Figure 6. Examples of (a) training mode, and (b) live mode of continuous breath encoding for the storage and the retrieval of breathing patterns linked to a user phrase using a mobile APP.

An early respiration activated AAC development involving a breath-to-text application was initiated at the Cavendish Laboratory at Cambridge University [52]. The study presented the use of fine breath tuning to use Dasher to support the communicative requirements of AAC users. Dasher is a text-entry system with a predictive language model available on several operating systems, and uses single and two-dimensional inputs from pointing devices to access an on-screen cursor. The fine breath tuning system encodes letters using Dasher's interface and a specially designed thoracic belt worn around the chest. Two inches of the belt are replaced by an elastic material, with a sensor measuring the changes of a user's waist circumference resulting from breathing variations. The study reports an expert user conversational rate of 15 words per minute using this system. The usage of sniffing signals was also established in the scope of AAC in [53]. A device was developed for the measurement of human nasal pressure via a nasal cannula and a pressure transducer. The device was tested with individuals in LIS, and quadriplegic users. To write text, the captured nasal pressure changes are converted into electrical signals, and passed to a computer. The device comprises two associated 
interfaces for the user's selection of letters, including a letter-board interface, and a cursor-based interface. The system aided the users in LIS, with reported rates of three letters per minute.

Microphones could be also used in combination of an AAC interface. The loss of speech abilities associated with SLCN centralizes the usage of microphones around two AAC areas, including speech augmentation of individuals suffering partial loss of speech [54] and breath encoding for individuals with speech disabilities [51,55]. Encoding distinct inhalation and exhalation signals was presented in [55] to produce synthesized machine spoken words (SMSW) through soft and heavy blows represented through four bit combinations of zeros and ones. The classification is achieved based on the threshold values of the generated blows. A micro-controller unit together with an MP3 voice module are appended to the microphone for the execution of the pattern classification and the playback of SMSW. The 16 discrete combinations were linked to predefined phrases selected with the aid of medical practitioners. "TALK" is also a solution involving a micro-electro-mechanical-system (MEMS) microphone together with two low-cost micro-controllers, and is similarly in use with distinct inhalation and exhalation signals to encode letters through the International Morse Code to produce SMSW [2]. A study has also reported the use of analogue breath encoding for AAC purposes by utilizing the recognition of continuous breathing modulations [51]. Analogue encoding of the acquired breathing signals was reported to provide an increased bandwidth at the low breathing frequencies, as it utilizes the signal's amplitude, frequency and phase changes to encode a user's intended meanings. The classification is achieved based on the dynamic time warped distances between the tested breathing patterns. A systematic reliability of $89 \%$ was reported with increased familiarity with the system.

\subsubsection{Brain Computer Interface Methods}

In the scope of $\mathrm{AAC}$, Brain Computer Interface (BCI) solutions are being widely researched to allow AAC users to control external devices by modulating their brain signals [56-58]. Brain interfaces are either invasive or non-invasive. Invasive interfaces involve the usage of implanted electrodes and the interconnections of the brain with the peripheral nerves [57]. Non-invasive BCIs comprise the usage of external devices to monitor a user's brain activities through EEG [54,57], magnetoencephalography (MEG) [56], functional magnetic resonance imaging (fMRI) [56,57] or near-infrared spectroscopy (NIRS) $[56,57]$. The components and flow diagram of a typical BCI system are shown in Figure 7.

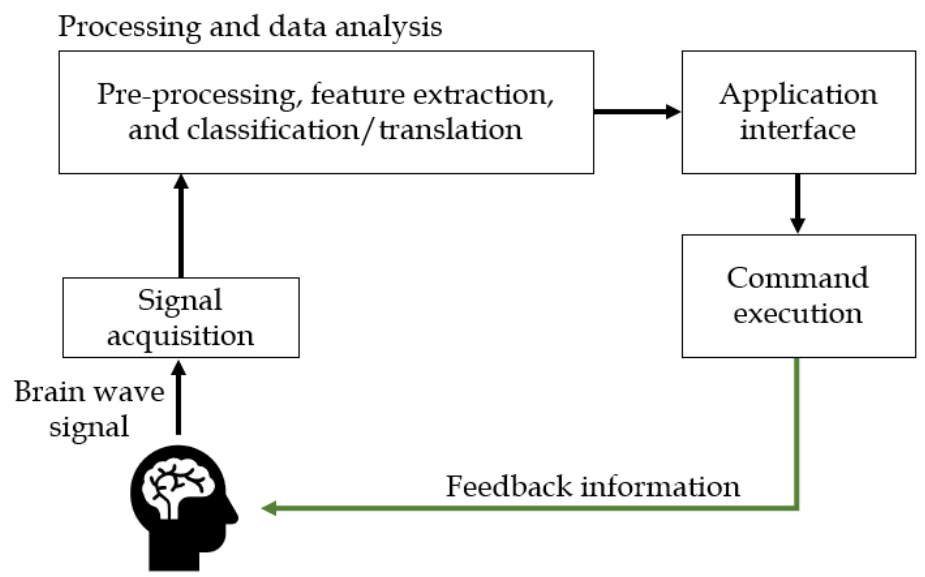

Figure 7. The components and flow diagram of a Brain Computer Interface (BCI) system, adapted from $[59,60]$.

EEG is a popular BCI recording method, given its non-invasive nature and its relatively lower cost [61,62]. In electrical BCI systems, the brain produces a set of electrical signals when triggered by a stimulus, known as the evoked potential [63]. EEG signals are acquired through two to 64 sensors placed on the scalp of the user to record the brain activity [64]. Amplifiers and filters are typically utilized, with an output fed back to the user to accordingly modulate the brain activity [57]. To translate 
a brain activity into a computer command, regression and classification algorithms could be used [65]. An adaptive auto-regressive (AR) parameter estimation model used with EEG BCI describes a time series signal $x(t)$ as

$$
\begin{gathered}
x(t)=\sum_{i=1}^{p} \phi_{i} x(t-i)+\epsilon_{t} \\
x(t)=\phi_{1} x(t-1)+\ldots+\phi_{p} x(t-p)+\epsilon_{t},
\end{gathered}
$$

where $\phi_{i}$ and $\mathrm{p}$ are the AR coefficients and the order of the model respectively, and $\epsilon_{t}$ is white noise [66,67]. A review study [65] demonstrates that the use of classification algorithms is an increasingly popular approach with BCI interfaces, as they are commonly used to identify the acquired brain patterns. Classification is the process of using a mapping $f$ to predict the correct label $y$ corresponding to a feature vector $x$. A training set $T$ is used with the classification model to find the best mapping $f^{*}$ [65]. The classification accuracy of a model is dependent on a variety of factors. A study [65] demonstrates that using the mean square error (MSE), three sources are identified to be the cause of classification errors, given that

$$
M S E=E\left[(y-f(x))^{2}\right]
$$

could be decomposed into

$$
M S E=\operatorname{Var}(f(x))+\operatorname{Bias}(f(x))^{2}+\sigma^{2},
$$

where the variance (Var) represents the model's sensitivity to $T$, the Bias represents the accuracy of the mapping $f$, and the noise $\sigma^{2}$ is the irreducible error present in the system. Common ML algorithms used with BCI include linear classifiers (such as linear support vector machines), neural networks, non-linear bayesian classifiers, nearest neighbors, and combinations of classifiers $[64,65]$. Signal processing techniques pertient to BCI methods include both time-frequency analysis, such as AR models, wavlets, and Kalman filtering, and spatiotemporal analysis, such as the Laplacian filter [68]. Hybrid BCI is a different approach to brain signals processing, combining a variety of brain and body signals in sequential and parallel processing operations with the aim of improving the accuracy of BCI systems [69].

$\mathrm{BCIs}$ are under continuous research to aid the communication of individuals suffering from motor strokes [56], ALS, and LIS, and spinal cord injuries [70]. BCI systems involve three basic pillars, including the user training, the associated ML, and the application in use [71,72]. Research in the area of BCIs is currently evolving [56], with promising results in recent state-of-the-art projects. A study by Stanford University [73] confirmed the usability of BCIs to control an unmodified smart device for quadriplegic users. BCIs have been also in use to surf the internet [74], with an EEG BCI based application tested with LIS and ALS conditions [75]. It is also reported that BCIs could aid users control spelling and play games [73].

\subsection{Machine and Deep Learning}

Typical signal processing of the acquired AAC signals encompasses three primary operations: encoding, prediction, and retrieval [10]. Encoding involves the conversion of the acquired signal into a pre-defined format accepted by the system for the production of a specified output, whereas prediction is concerned with building the algorithms used to select the desired output [10]. Prediction encompasses several operational contexts, including word [76], message, and icon prediction [10]. In general, an ideal AAC system should integrate self-learning capabilities to respond to its users' individual needs $[2,8]$. Demographic data shows that current AAC users belong to numerous cultural and linguistic backgrounds [7]. In turn, the design of systems tailored to address specific users' requirements is vital. High-tech AAC is hence becoming a highly interdisciplinary area of research, combining rehabilitation engineering with clinical and psychological studies, signal processing, and ML [77]. 
ML has been widely evolving over the last decade, with a number of applications aimed at aiding the provision of intelligent AAC solutions to address the users' needs. The automation of algorithms, prediction, and classification capabilities presented by ML solutions could be of great benefit to the users. Technologies such as natural language processing (NLP) are highly dependent on artificial intelligence (AI). The operation of NLP is centered around the analysis, augmentation, and generation of language, including the computation of probabilities of incoming words and phrases, and complete sentence transformations [78]. NLP has various applications in AAC, utilizing ML and statistical language models to process and generate outputs by optimizing word prediction models, topic models [79], speech recognition algorithms, and processing of the context of usage [78]. BCI is also highly dependent on $\mathrm{ML}$, as users learn to encode the desired intended messages through dedicated brain signal features captured by the BCI for the translation to the intended meaning or the desired control $[71,77,80,81]$. Recent studies also show that advances with DL algorithms, such as conventional and recurrent neural networks, could have a potential superior performance in comparison with conventional classification methods [63].

\subsection{Outputs and Speech generating Devices}

High-tech AAC systems can produce outputs in a variety of formats, including symbols, icons, and electronic digitized or synthesized speech [10]. SGDs, or voice output communication aids (VOCAs), are devices with the ability to produce digitized or synthesized speech $[9,82]$. Digitized speech is pre-stored speech acquired via a microphone and stored in electronic format for retrieval upon a user action [83]; whereas synthesized speech is generated based on mathematical algorithms and played as natural voice [10]. The wide availability of smart devices facilitates the access to VOCA applications. Synthesized speech includes the production of output messages via text to speech synthesis, and is therefore commonly researched to assist the communication and free personal expression of speech impaired individuals. This is primarily due to the benefit of providing a greater flexibility in contrast with digitized speech. Studies show that AAC devices with SGD capabilities contribute to significant developments in terms of AAC solutions [10,82]. However, the efficiency and effectiveness of using a VOCA with an AAC user remains dependent on the user's abilities, their medical condition, and the communication partners they are conversing with [9].

\section{Comparison of Existing AAC Signal Sensing Methods}

With respect to the discussed HAAT model and the listed AAC access methods, the integration of state-of-the-art AAC systems with AI applications could help in the improvement and the ease of use of common AAC devices and their associated user interfaces. The focus on the user activity to be carried out needs to be at the core of the implementation. Table 2 provides a comparison of the input signal sources, the requirements for operation, the areas of strength, and the areas of limitation of the listed AAC sensing methods. A summary of each of the listed access categories is demonstrated below in terms of the ease of access, affordability, ease of programming and maintaining, portability, and conversational rates.

\subsection{Ease of Access}

Imaging methods, including eye gaze and eye tracking methods, are generally utilized as non-invasive means of communication for the individuals with minimal voluntary controls and motor movements. The natural eye gazing process is a an advantageous trait for accessing devices [26]. However, typical imaging methods were shown to require a learning curve for both the users and the systems, as a calibration step is usually required for the customization of an imaging device to each individual user [84]. The accuracy of the system is also dependent on many variants, including the gaze angle, the pixel accuracy, and the distance between the eye and the screen, as demonstrated in equations (1), (4), and (5), rendering eye gaze difficult when selecting small items on the screen [26]. The movements of the head and the direction of the gaze might impact the usability of the acquired 
signals, usually with algorithms implemented to cancel out the effects of such movements [22]. Recent eye gaze systems are better proofed against head movements, and in turn need to be calibrated less frequently [54]. Calibration models are also in use to facilitate the process of gaze calibration [85]. Recent studies are beginning to address the current constraints to create forthcoming robust imaging systems that are easy to use [84]. Mechanical and electro-mechanical activated switches and keyboards are usually easier to operate due to their simplistic nature. Mechanical switches are predominantly used with individuals requiring minimal motor movements to access a computer or a smart device via indirect selection [4]. Touch-activated methods require voluntary muscle controls, however with a minimal activation pressure, as discussed in Table 2. Touchscreens could be also used in combination with mechanical switches for individuals lacking motor controls to access the devices for indirect selections enablement. This multi-modal access can in turn be advantageous, as users will have a choice to access the device using more than one modality. Breath-activated methods are similarly used with individuals with minimal voluntary controls; however, they require a training step to recognize the selected patterns [51,55]. With regards to BCI access, non-invasive methods, such as EEG, are used due to their non-intrusive nature. BCI systems provide a natural means of access, aiding the users to gain independence [86]. However, the signal acquisition from the brain is at times cumbersome for the users, especially with EEG applications requiring the use of electrolytic gel to facilitate the acquisition of the brain signals from the scalp [87]. The length of the training process required to use a BCI system could also present a challenge for usage [88].

\subsection{Affordability}

In terms of costs, the expenses associated with the hardware and software requirements of the utilized platforms directly impact the expenses related to the systems. Imaging methods, including eye gazing and tracking, are relatively expensive in comparison to switch access, touch based methods, and breath-activated methods. This is mainly due to the high-costs associated with the systems' hardware requirements, which are listed in Table 2, together with the costs of research, programming and maintaining the devices [89]. Depending on a solution's capabilities, the price of a typical eye tracker ranges between hundreds to thousands of dollars [84]. Some solutions are emerging to reduce the costs of imaging AAC devices [27,84]; however, more research is still needed to widen the scope of usage of highly performing, low-cost eye trackers. On the other hand, mechanical keyboards and access switches are commonly simple to design and thus they are usually more affordable. The reliance of switch access or touch-based methods on a smart or a high-tech dedicated device could increase the costs of the provided solutions; however, with the prevalence of smart devices, several AAC communicative applications (APPs) are now available on various operating systems, widening the usability of AAC in contrast with traditional SGDs [16]. As listed in Table 2, breath-activated methods are usually accessed using pressure sensors or microphones together with micro-controller boards or a computer. The hardware requirements could increase the costs of the solutions; however, the escalating prevalence of smart devices might aid in the provision of cost-effective breath-activated APPs. BCI methods are also being researched to reduce the costs associated with the systems [69]; however, low-cost BCI systems were reported to require further research to improve the accuracy and quality of the acquisition in comparison with advanced BCI systems.

\subsection{Ease of Programming and Maintaining}

Programming an AAC access modality is dependent on the acquired signals, together with the research and skills required to set up and maintain the systems. Typical imaging devices are associated with increased complexities in terms of algorithm writing, data processing, and data parsing $[90,91]$. This in turn requires extensive programming and coding skills to set up the gaze detection algorithms, calibrate the sensors to individual users, and accurately respond to the needs of the users. The resulting amount of data also needs to be addressed, with specific considerations to 
sample sizes and data resolution [91]. Mechanical switch access of smart devices, and the programming of touch-activated APPs is in turn less variant in terms of calibration and set up. The APPs however need to be carefully designed and tested to respond to the user's input generated via a switch, a keyboard, or a touchscreen. Touch activated methods also need to incorporate a visual or auditory feedback mechanism to confirm the user's selection, as demonstrated in Table 2 [19]. Breath-activated methods are similarly programmed based on breath thresholds [55], and classification algorithms [51]. The complexities are in turn dependent on the requirements of the APP design together with the selected classification algorithms required for the system operation. Concerning BCI methods, the challenges related to managing and programming the systems are centred around the information transfer rates, the non-linearity of the systems, and the complexities associated with the high signals' dimensionalities [88].

\subsection{Portability}

In terms of portability, the typical requirements of the systems dictate the ease of moving the device for usage in a different setting. Commercial solutions of the AAC imaging methods are starting to address this constraint to increase the usability of the devices [42,84]; however, most typical system requirements still restrict eye-tracking systems to be used indoors [23] or together with a monitor. On a similar note, the portability of mechanically activated switches is variant depending on the context of usage; however, the integration of switch access with mobile and smart devices increases the ease of portability. Touch-activated methods are similarly highly portable, given the typical sizes of the smart devices used in coordination with the method. On the other hand, the portability of breath-activated systems is application dependent, as the solutions requiring the need of a computer interface still need to be developed to address this constraint. BCI methods are still challenged in terms of the communicative interfaces [88]; however, some advances in BCI have been reported for the potential possibility of home usage [86] and increased portability [92].

\subsection{Conversational Rates}

Natural speech has a rate of 125 to 185 words per minute (WPM) [93]. Speech rates of less than 100 WPM are identified as slow [94]. Direct selection techniques, including eye gaze systems, are found to provide conversational rates of about 8-10 WPM [93]. Likewise, mechanically activated AAC switches and keyboards also affect the conversational rates. The automatic, step, and inverse activation of switches often requires the users to wait until the desired selection is displayed, introducing conversational delays. Scanning methods were reported to allow communicative rates of around two WPM [93]. Selecting letters to form words may also impact the user's communication rate. This is apparent in touch-activated methods, where users are required to spell words or select icons to form sentences or to write text. The conversational rates of breath-activated systems are further dependent on the encoding method, as systems where breathing variations are used to select letters to write words could negatively impact the conversational rates. A recent study [87] similarly reported that most BCI technologies still offer conversational rates of less than 20 letters per minute. Generally, the rates of conversation using AAC systems, including word prediction and letter abbreviation, were found to be between 12-18 WPM, highly contrasting with the rates of natural speech [93]. 
Table 2. Signal sources, areas of strength and areas of limitation of current commercial AAC devices

\begin{tabular}{|c|c|c|c|c|}
\hline Signal Source & Mode & Typical Hardware Requirements & Areas of Strength & Limitations and Areas of Improvement \\
\hline \multirow[t]{2}{*}{ Imaging methods } & Eye gazing & $\begin{array}{l}\text { - IR/NIR illumination source } \\
\text { (commonly: } 850+/-30 \mathrm{~nm} \text { ) } \\
\text { - Monitor } \\
\text { - Camera }\end{array}$ & $\begin{array}{l}\text { - Non-invasive } \\
\text { - Minimal voluntary control of muscles } \\
\text { - Can be used with patients requiring } \\
\text { mechanical ventilation [26] } \\
\text { - IR is invisible to the user's eyes[23] } \\
\text { - IR can stabilize gaze estimation [23] }\end{array}$ & $\begin{array}{l}\text { - High Temporal resolution }=\text { high volume } \\
\text { of data as patterns are averaged over long } \\
\text { time spans [90]; Consequence: sample sizes } \\
\text { are often small [91] } \\
\text { - Eye tracking data processing [91] and } \\
\text { parsing is complex [90] } \\
\text { - Need for calibration algorithms } \\
\text { - IR signals are not reliable for outdoor use [23] } \\
\text { - Generally, high cost [27] }\end{array}$ \\
\hline & Head-pointing & $\begin{array}{l}\text { - Head-mounted visors } \\
\text { in addition to a monitor } \\
\text { and a camera [95] } \\
\text { - Light/optical pointers }[4,19]\end{array}$ & $\begin{array}{l}\text { Less expensive compared to typical } \\
\text { eye-gaze systems }\end{array}$ & $\begin{array}{l}\text { - Need fine user precision and controls [95] } \\
\text { - In direct contact with the user's head }\end{array}$ \\
\hline \multirow{2}{*}{$\begin{array}{l}\text { Mechanical/Electro- } \\
\text { mechanical } \\
\text { methods }\end{array}$} & $\begin{array}{l}\text { Automatic, step, or } \\
\text { inverse activation }\end{array}$ & $\begin{array}{l}\text { Single switch, } \\
\text { array of switches }\end{array}$ & $\begin{array}{l}\text { Requires minimal motor control } \\
\text { [4] }\end{array}$ & Generally slow \\
\hline & $\begin{array}{l}\text { Typing/icon } \\
\text { selection }\end{array}$ & Mechanical keyboards & $\begin{array}{l}\text { Instant feedback to user } \\
\text { whenever a key is pressed } \\
{[4,19]}\end{array}$ & $\begin{array}{l}\text { Voluntary muscle control is } \\
\text { a requirement for activation } \\
{[4,19]}\end{array}$ \\
\hline $\begin{array}{l}\text { Touch-activated } \\
\text { methods }\end{array}$ & $\begin{array}{l}\text { Force production } \\
\text { through: } \\
\text { - Hand/arm/ } \\
\text { body part control } \\
\text { - control extender } \\
\text { [96] }\end{array}$ & $\begin{array}{l}\text { - Resistive/Capacitive } \\
\text { touch screen circuitry } \\
\text { - Membrane keyboards: } \\
\text { Non-conductive spacers } \\
\text { separating conductive flat } \\
\text { surfaces } \\
{[4,19]}\end{array}$ & Minimal activation pressure & $\begin{array}{l}\text { - No direct feedback upon activation } \\
\text { - Requires appended feedback } \\
\text { mechanisms (auditory/sensory) } \\
{[4,19]}\end{array}$ \\
\hline
\end{tabular}


Table 2 (Continued). Signal sources, areas of strength and areas of limitation of current commercial AAC devices

\begin{tabular}{|c|c|c|c|c|}
\hline Signal Source & Mode & Typical Hardware Requirements & Areas of Strength & Limitations and Areas of Improvement \\
\hline \multirow[t]{4}{*}{$\begin{array}{l}\text { Breath-activated } \\
\text { methods }\end{array}$} & $\begin{array}{l}\text { Fine breath } \\
\text { tuning [52] }\end{array}$ & $\begin{array}{l}\text { - Thoracic belt } \\
\text { - Sensor measuring the changes } \\
\text { of waist circumference }\end{array}$ & $\begin{array}{l}\text { Integration with a } \\
\text { predictive language model }\end{array}$ & $\begin{array}{l}\text { - Physical control of movements: } \\
\text { restricted for paralysed users. } \\
\text { - Portability constraints } \\
\text { - Slow conversational rate }\end{array}$ \\
\hline & $\begin{array}{l}\text { Sniff control } \\
\text { [53] }\end{array}$ & $\begin{array}{l}\text { Control sensors for the acquisition } \\
\text { of nasal pressure. }\end{array}$ & $\begin{array}{l}\text { Confirmed usability with } \\
\text { patients in LIS }\end{array}$ & $\begin{array}{l}\text { Slowness: rate of } \\
\text { three characters per minute. }\end{array}$ \\
\hline & $\begin{array}{l}\text { Discrete } \\
\text { breath encoding } \\
{[55,97]}\end{array}$ & $\begin{array}{l}\text { - Microphones/MEMS sensors } \\
\text { - Microcontroller boards }\end{array}$ & Wearable configuration & $\begin{array}{l}\text { - Digitized inputs } \\
\text { - Predefined words and sentences } \\
\text { (not user-selected) } \\
\text { - Confinement to limited patterns. }\end{array}$ \\
\hline & $\begin{array}{l}\text { Analogue breath } \\
\text { encoding [51] }\end{array}$ & $\begin{array}{l}\text { - Microphone } \\
\text { - PC }\end{array}$ & Continuous/analogue breath encoding & $\begin{array}{l}\text { - The processing of warped distances } \\
\text { is computationally complex } \\
\text { - Portability constraints }\end{array}$ \\
\hline \multirow[t]{2}{*}{ BCI methods } & Invasive & Implantable electrodes & \multirow{2}{*}{$\begin{array}{l}\text { Communication and control of } \\
\text { environment without the need } \\
\text { for body movements } \\
\text { [57] }\end{array}$} & \multirow{2}{*}{$\begin{array}{l}\text { - Prone to classification errors [22] } \\
\text { - Low transfer rates of ECG-based BCI } \\
\text { due to the low signal to noise ratio [61] } \\
\text { - Most platforms are not yet suitable } \\
\text { for everyday usage/ in-home usage } \\
\text { - BCI devices often require extensive } \\
\text { assistance from caregivers [98] }\end{array}$} \\
\hline & Non-invasive & $\begin{array}{l}\text { External monitoring: EEG, } \\
\text { MEG, fMRI, NIRS. } \\
{[56]}\end{array}$ & & \\
\hline
\end{tabular}




\section{Prospective Advances in Future AAC}

Studies are currently directed towards the establishment of intelligent AAC systems that are suited to respond to personal users' needs, intended activities, and individual capabilities $[2,51,55]$. The development of robust future AAC solutions should hence take into consideration some of the shortfalls of the current technologies. The conversational rates of most available AAC solutions are still slow, offering a rate of around 12-18 WPM in contrast with that of natural speech (125-185 WPM). $[76,93]$. In turn, AAC users still communicate at a rate that is approximately $10 \%$ the rate of natural speech [99]. This is most apparent in text-based communication involving complex navigation to search or select specific messages or to type full sentences to convey a meaning. On a similar note, the users are sometimes unable to solely rely on the devices due to the limited range of available words / phrases, in turn only engaging in "routinized conversations" using their AAC equipment [8]. As implied from Table 2, such conditions are further complicated with the increased complexities of some AAC systems, which might require special support from the user's carers to set up and operate the systems. The access and operation of AAC devices with minimal user movements is also vital, as the generation of voluntary movements may be cumbersome for users suffering extreme forms of paralysis.

In light of the discussed AAC sensing and acquisition modalities and the AT requirements reviewed using the HAAT model, the following implications can be drawn:

\section{(A) Affordability}

One of the barriers to the realization of the full-potential of high-tech AAC systems is related to the affordability of the devices. The expenses associated with speech generation, together with the hardware and processing requirements of most high-tech AAC sensing modalities, hinder the scale at which high-tech AAC systems are used, and in consequence, negatively impacting the scale at which high-tech AAC is expanding. In turn, low-technology AAC remains widely utilized by speech and language therapists, given its affordability and wide abundance. Therefore, addressing the affordability of high-tech AAC platforms is a necessity for high-tech AAC systems to become prevalent.

\section{(B) Mobile APP integration}

The development of AAC APPs utilizing the capabilities of smart phones and tablets can be further explored to assist the communication of speech impaired individuals, in turn reducing cost and enhancing portability. As demonstrated, the usage of VOCAs is of a potential benefit for users of different age ranges suffering a variety of medical conditions [100]. However, it is shown that the variability in terms of operational principles, user groups, and the complexity of a real and complicated user's environment still need to be tackled. The integration of smart devices and VOCA APPs could be researched to expand the scope of high-tech assisted voice communication.

\section{(C) DL functionalities}

AAC methods incorporating analogue encoded signals via the acquisition of continuous user inputs are generally less cumbersome to generate in comparison with discrete encoding. For example, the discretization process of inputting letters to form sentences via imaging, touch-activated, or breath-activated methods may slow down a user's conversational rate due to the efforts required to navigate the boards of letters to construct the intended phrases. The integration of state-of-the-art AAC systems with AI and DL applications can be researched to improve the access to high-tech devices, the speed of the output generation and the customization of the AAC interface to suit each individual user.

\section{(D) NLP and intelligent AAC}

By comparing the listed high-tech AAC sensing modalities, a trade-off is apparent between the speed of conversing, and the free expression of the user. Pre-programmed phrases restrict the dynamicity of the user's conversations; whereas spelling based communication is generally slow. Further research 
469

\section{Abbreviations} not-for-profit sectors.

with regards to NLP and DL functionalities is needed for the provision of innovative activity-oriented AAC methods to support the user, the facilitator, and the communication partner [93].

\section{(E) Forthcoming development of mobile health applications}

The implications for future research could expand beyond the usage of the AAC devices for simple communication, as the integration of high-tech AAC with accessible smart devices paves the way for state-of-the-art developments, such as mobile health (m-Health) communicative applications, to exist. The development of smart mobile platforms would in turn aid the remote communication between users and their medical practitioners. This will expand the scope of AAC beyond physical communications, increasing the usability and the context of usage of future AAC solutions.

Author Contributions: Y.E. compared the reviewed methods and wrote the manuscript. S.H. structured and organized the manuscript, and supervised the review writing. D.K. and K.B.-M. guided the analysis and revised the manuscript, and A.M. engaged in the analysis of the breath-activated methods

Funding: This research received no specific grant from any funding agency in the public, commercial, or

Acknowledgments: The authors would like to acknowledge the support of Loughborough University in the conduction of this review.

Conflicts of Interest: The authors declare no conflict of interest.

The following abbreviations are used in this manuscript:

AAC augmentative and alternative communication

AI artificial intelligence

ALS Amyotrophic Lateral Sclerosis

APP applications

AR auto-regressive

ASD Autistic Spectrum Disorders

AT assistive technology

$\mathrm{BCI}$ brain computer interface

DL deep learning

EEG electroencephalogram

fMRI functional magnetic resonance imaging

HAAT Human Activity Assistive Technology

IR Infrared

IR-PCR Infrared pupil corneal reflection

489 LIS

MEG

MEMS micro-electro-mechanical systems

ML machine learning

MSE mean square error

NIR near-infrared

NIRS near-infrared spectroscopy

NLP natural language processing

PPG photoplethysmogram

SGD speech generating device

SLCN speech, language or communication need

SMSW synthesized machine spoken words

VOCA voice output communication aid

WPM words per minute 


\section{References}

1. García-Méndez, S.; Fernández-Gavilanes, M.; Costa-Montenegro, E.; Juncal-Martínez, J.; Javier González-Castaño, F. Automatic natural language generation applied to alternative and augmentative communication for online video content services using simple NLG for Spanish. In Proceedings of the Proceedings of the 15th Web for All Conference: Internet of Accessible Things, W4A 2018; Lyon, France, 2018.

2. Kerr, D.; Bouazza-Marouf, K.; Gaur, A.; Sutton, A.; Green, R. A breath controlled AAC system. In Proceedings of the CM2016 National AAC Conference, Orlando, FL, USA, 19-22 April 2016; pp. 11-13.

3. Schultz Ascari, R.E.O.; Pereira, R.; Silva, L. Mobile Interaction for Augmentative and Alternative Communication: a Systematic Mapping. SBC J. Interact. Syst. 2018, 9, 105-118.

4. M. Cook, A.; Polgar, J.M. Assistive Technologies Principles and Practices; 4th ed.; Elsevier: USA, 2015;

5. Smith, A. Speech motor development: Integrating muscles, movements, and linguistic units. J. Commun. Disord. 2006, 39, 331-349.

6. van de Sandt-Koenderman, M.W.M.E. High-tech AAC and aphasia: Widening horizons? Aphasiology 2004, 18, 245-263.

7. Light, J.; McNaughton, D. The Changing Face of Augmentative and Alternative Communication: Past, Present, and Future Challenges. Augment. Altern. Commun. 2012, 28, 197-204.

8. Hodge, S. Why is the potential of augmentative and alternative communication not being realized? Exploring the experiences of people who use communication aids. Disabil. Soc. 2007, 22, 457-471.

9. Mirenda, P. Toward Functional Augmentative and Alternative Communication for Students With Autism. Lang. Speech Hear. Serv. Sch. 2003, 34, 203.

10. National Academies of Sciences, Engineering, and Medicine. Augmentative and Alternative Communication and Voice Products and Technologies. In The promise of assistive technology to enhance activity and work participation; The National Academies Press: Washington, DC, 2017; pp. 209-273.

11. Smith, E.; Delargy, M. Locked-in syndrome. Br. Med. J. 2005, 330, 406-409.

12. Simion, E. Augmentative and Alternative Communication - Support for People with Severe Speech Disorders.Procedia - Soc. Behav. Sci. 2014, 128, 77-81.

13. Arthanat, S.; Bauer, S.M.; Lenker, J.A.; Nochajski, S.M.; Wu, Y.W.B. Conceptualization and measurement of assistive technology usability. Disabil. Rehabil. Assist. Technol. 2007, 2, 235-248.

14. Giesbrecht, E. Application of the human activity assistive technology model for occupational therapy research. Aust. Occup. Ther. J. 2013, 60, 230-240.

15. Iacono, T.; Lyon, K.; Johnson, H.; West, D. Experiences of adults with complex communication needs receiving and using low tech AAC: an Australian context. Disabil. Rehabil. Assist. Technol. 2013, 8, 392-401.

16. McNaughton, D.; Light, J. The iPad and mobile technology revolution: Benefits and challenges for individuals who require augmentative and alternative communication. AAC Augment. Altern. Commun. 2013, 29, 107-116.

17. Shane, H.C.; Blackstone, S.; Vanderheiden, G.; Williams, M.; Deruyter, F. Using AAC technology to access the world. Assist. Technol. 2012, 24, 3-13.

18. Baxter, S.; Enderby, P.; Evans, P.; Judge, S. Barriers and facilitators to the use of high-technology augmentative and alternative communication devices: A systematic review and qualitative synthesis. Int. J. Lang. Commun. Disord. 2012, 47, 115-129.

19. Glennen, S.L. Augmentative and alternative communication systems. In The handbook of augmentative and alternative communication; 1997; pp. 59-69 ISBN 9780323096317.

20. Tobii Dynavox PCEye Plus Available online: https://www.tobiidynavox.com/en-gb/devices/eye-gaze -devices/pceye-plus-access-windows-control/\#Specifications (accessed on Feb 10, 2019).

21. Chennamma, H.R.; Yuan, X. A Survey on Eye-Gaze Tracking Techniques. Indian J. Comput. Sci. Eng. 2013, 4, 388-393.

22. Kar, A.; Corcoran, P. A review and analysis of eye-gaze estimation systems, algorithms and performance evaluation methods in consumer platforms. IEEE Access 2017, 5, 16495-16519.

23. Hansen, D.W.; Ji, Q. In the Eye of the Beholder: A Survey of Models for Eyes and Gaze. IEEE Trans. Pattern Anal. Mach. Intell. 2010, 32, 478-500. 
24. Townend, G.S.; Marschik, P.B.; Smeets, E.; van de Berg, R.; van den Berg, M.; Curfs, L.M.G. Eye Gaze Technology as a Form of Augmentative and Alternative Communication for Individuals with Rett Syndrome: Experiences of Families in The Netherlands. J. Dev. Phys. Disabil. 2016, 28, 101-112.

25. Chen, S.-H.K.; O'Leary, M. Eye Gaze 101: What Speech-Language Pathologists Should Know About Selecting Eye Gaze Augmentative and Alternative Communication Systems. Perspect. ASHA Spec. Interes. Groups 2018, 3, 24-32.

26. Ball, L.; Nordness, A.; Fager, S.; Kersch, K.; Mohr, B.; L. Pattee, G.; Beukelman, D. Eye-Gaze Access to AAC Technology for People with Amyotrophic Lateral Sclerosis. J. Med. Speech. Lang. Pathol. 2010, 18, 11-23.

27. Corno, F.; Farinetti, L.; Signorile, I.; Torino, P. A Cost-effective solution for eye-gaze assistive technology. In Proceedings of IEEE International Conference on Multimedia and Expo; IEEE: Lausanne, Switzerland, Switzerland, 2002; pp. 433-436.

28. Majaranta, P.; Aoki, H.; Donegan, M.; Hansen, D.W.; Hansen, J.P. Gaze Interaction and Applications of Eye Tracking: Advances in Assistive Technologies; IGI Publishing: Hershey, PA, 2011.

29. Bates, R.; Donegan, M.; Istance, H.O.; Hansen, J.P.; Räihä, K.J. Introducing COGAIN: Communication by gaze interaction. Univers. Access Inf. Soc. 2007, 6, 159-166.

30. Bates, R.; Istance, H.; Oosthuizen, L.; Majaranta, P. Survey of De-Facto Standards in Eye Tracking. COGAIN Commun. by gaze Interact. 2005.

31. Al-Rahayfeh, A.; Faezipour, M. Eye Tracking and Head Movement Detection: A State-of-Art Survey. IEEE J. Transl. Eng. Heal. Med. 2013, 1, 2100212.

32. Janthanasub, V. Ophapasai: Augmentative and Alternative Communication Based on Video-Oculography Control Interface. Appl. Mech. Mater. 2016, 848, 60-63.

33. Tai, K.; Blain, S.; Chau, T. A Review of Emerging Access Technologies for Individuals With Severe Motor Impairments. Assist. Technol. 2008, 20, 204-221.

34. Harezlak, K.; Kasprowski, P. Application of eye tracking in medicine: A survey, research issues and challenges. Comput. Med. Imaging Graph. 2018, 65, 176-190.

35. van der Geest, J.N.; Frens, M.A. Recording eye movements with video-oculography and scleral search coils: a direct comparison of two methods. J. Neurosci. Methods 2002, 114, 185-195.

36. Robinsont, D. A Method of Measuring Eye Movement Using a Scleral Search Coil in a Magnetic Field IEEE Trans. Bio-Medical Electron. 1963, 10, 137-145.

37. Tobii Technology Accuracy and precision test method for remote eye trackers - Test Specification Report; 2011.

38. Farivar, R.; Michaud-Landry, D. Construction and Operation of a High-Speed, High-Precision Eye Tracker for Tight Stimulus Synchronization and Real-Time Gaze Monitoring in Human and Animal Subjects. Front. Syst. Neurosci. 2016, 10(73), 1-10.

39. Schwiegerling, J.T. Eye Axes and Their Relevance to Alignment of Corneal Refractive Procedures. J. Refract. Surg. 2013, 29, 515-516.

40. Salvucci, D.D.; Goldberg, J.H. Identifying fixations and saccades in eye-tracking protocols. In Proceedings of the Proceedings of the symposium on Eye tracking research \& applications. Florida, USA, 2000; pp. 71-78.

41. Poole, A.; Ball, L.J. Eye Tracking in Human-Computer Interaction and Usability Research: Current Status and Future Prospects.

42. Kunka, B.; Kostek, B. Non-intrusive infrared-free eye tracking method. In Proceedings of the Signal Processing Algorithms, Architectures, Arrangements, and Applications Conference Proceedings (SPA), 2009; Poznan, Poland, 2009; pp. 105-109.

43. MacKenzie, I.S.; Ashtiani, B. BlinkWrite: Efficient text entry using eye blinks. Univers. Access Inf. Soc. 2011, 10, 69-80.

44. Bhalla, M.R.; Bhalla, A.V. Comparative Study of Various Touchscreen Technologies. Int. J. Comput. Appl. 2010, 6, 12-18.

45. Lee, D. The State of the Touch-Screen Panel Market in 2011. Inf Disp 2011, 27, 12-16.

46. Qin, H.; Cai, Y.; Dong, J.; Lee, Y.-S. Direct Printing of Capacitive Touch Sensors on Flexible Substrates by Additive E-Jet Printing With Silver Nanoinks. J. Manuf. Sci. Eng. 2017, 139, 31011.

47. Massaroni, C.; Venanzi, C.; Silvatti, A.; Lo Presti, D.; Saccomandi, P.; Formica, D.; Giurazza, F.; Caponero, M.; Schena, E. Smart textile for respiratory monitoring and thoraco-abdominal motion pattern evaluation. J. Biophotonics 2018. 
48. Itasaka, Y.; Miyazaki, S.; Tanaka, T.; Shibata, Y.; Ishikawa, K. Detection of Respiratory Events during Polysomnography-Nasal-Oral Pressure Sensor Versus Thermocouple Airflow Sensor. Pract. Oto-Rhino-Laryngol. 2010, 129, 60-63.

49. Zhang, X.; Ding, Q. Respiratory rate monitoring from the photoplethysmogram via sparse signal reconstruction. Physiol. Meas. 2016, 37, 1105-1119.

50. Yahya, O.; Faezipour, M. Automatic detection and classification of acoustic breathing cycles. In Proceedings of the 2014 Zone 1 Conference of the American Society for Engineering Education, Bridgeport, CT, USA, 3-5 April 2014.

51. Elsahar, Y.; Bouazza-Marouf, K.; Kerr, D.; Gaur, A.; Kaushik, V.; Hu, S. Breathing pattern interpretation as an alternative and effective voice communication solution. Biosensors 2018, 8, 1-10.

52. Shorrock, T.; MacKay, D.; Ball, C. Efficient Communication by Breathing. In Deterministic and Statistical Methods in Machine Learning; Springer: Heidelberg/Berlin, Germany, 2005; pp. 88-97.

53. Plotkin, A.; Sela, L.; Weissbrod, A.; Kahana, R.; Haviv, L.; Yeshurun, Y.; Soroker, N.; Sobel, N. Sniffing enables communication and environmental control for the severely disabled. Proc. Natl. Acad. Sci. USA 2010, 107, 14413-14418.

54. Fager, S.; Bardach, L.; Russell, S.; Higginbotham, J. Access to augmentative and alternative communication: New technologies and clinical decision-making. J. Pediatr. Rehabil. Med. 2012, 5, 53-61.

55. Garcia, R.G.; Ibarra, J.B.G.; Paglinawan, C.C.; Paglinawan, A.C.; Valiente, L.; Sejera, M.M.; Bernal, M. V.; Cortinas, W.J.; Dave, J.M.; Villegas, M.C. Wearable augmentative and alternative communication device for paralysis victims using Brute Force Algorithm for pattern recognition. IEEE 9th Int. Conf. Humanoid, Nanotechnology, Inf. Technol. Commun. Control. Environ. Manag. 2017, 1-6.

56. Chaudhary, U.; Birbaumer, N.; Curado, M.R. Brain-Machine Interface (BMI) in paralysis. Ann. Phys. Rehabil. Med. 2015, 58, 9-13.

57. Birbaumer, N.; Murguialday, A.R.; Cohen, L. Brain-computer interface in paralysis. Curr. Opin. Neurol. 2008, 21, 634-638.

58. Yeo, M.; Jiang, L.; Tham, E.; Xiong, W. Evaluation of a low-cost alternative communication device with brain control. In Proceedings of the Proceedings of the 2015 10th IEEE Conference on Industrial Electronics and Applications, ICIEA 2015; IEEE: Auckland, New Zealand, 2015; pp. 229-232.

59. Kaiser, V.; Bauernfeind, G.; Kreilinger, A.; Kaufmann, T.; Kübler, A.; Neuper, C.; Müller-Putz, G.R. Cortical effects of user training in a motor imagery based brain-computer interface measured by fNIRS and EEG. Neuroimage 2014, 85, 432-444.

60. Hippe, Z.S.; Kulikowski, J.L.; Mroczek, T.; Wtorek, J. A Robust Asynchronous SSVEP Brain-Computer Interface Based On Cluster Analysis of Canonical Correlation Coefficients. Adv. Intell. Syst. Comput. 2014, 300,3-14.

61. Chen, X.; Wang, Y.; Nakanishi, M.; Gao, X.; Jung, T.-P.; Gao, S. High-speed spelling with a noninvasive brain-computer interface. Proc. Natl. Acad. Sci. 2015, 112, E6058-E6067.

62. Tan, P.; Tan, G.; Cai, Z. Dual-tree complex wavelet transform-based feature extraction for brain computer interface. In Proceedings of the 12th International Conference on Fuzzy Systems and Knowledge Discovery, FSKD 2015; IEEE, 2015; pp. 1136-1140.

63. Thomas, J.; Maszczyk, T.; Sinha, N.; Kluge, T.; Dauwels, J. Deep learning-based classification for brain-computer interfaces. In Proceedings of the 2017 IEEE International Conference on Systems, Man, and Cybernetics, SMC 2017; 2017; Vol. 2017-Jan, pp. 234-239.

64. Gupta, A.; Parameswaran, S.; Lee, C.H. Classification of electroencephalography (EEG) signals for different mental activities using Kullback Leibler (KL) divergence. In Proceedings of the ICASSP, IEEE International Conference on Acoustics, Speech and Signal Processing; IEEE, Taipei, Taiwan, 2009; pp. 1697-1700.

65. Lotte, F.; Congedo, M.; Lécuyer, A.; Lamarche, F.; Arnaldi, B. A review of classification algorithms for EEG-based brain-computer interfaces. J. Neural Eng. 2007, 4.

66. Zhang, Y.; Ji, X.; Zhang, Y. Classification of EEG signals based on AR model and approximate entropy. In 2015 Proc. Int. Jt. Conf. Neural Networks, Killarney, Ireland, 2015-September.

67. Guger, C.; Schlögl, A.; Neuper, C.; Walterspacher, D.; Strain, T.; Pfurtscheller, G. Rapid prototyping of an EEG-based brain-computer interface (BCI). IEEE Trans. Neural Syst. Rehabil. Eng. 2001, 9, 49-58.

68. Ortiz-Rosario, A.; Adeli, H. Brain-computer interface technologies: from signal to action. Rev. Neurosci. 2013, 24. 
69. Choi, B.; Jo, S. A Low-Cost EEG System-Based Hybrid Brain-Computer Interface for Humanoid Robot Navigation and Recognition. PLoS One 2013, 8.

70. Nijboer, F.; Plass-Oude Bos, D.; Blokland, Y.; van Wijk, R.; Farquhar, J. Design requirements and potential target users for brain-computer interfaces-recommendations from rehabilitation professionals. Brain-Computer Interfaces 2014, 1, 50-61.

71. McFarland, D.J.; Wolpaw, J.R. Brain-computer interface use is a skill that user and system acquire together. PLoS Biol. 2018, 16, 10-13.

72. Perdikis, S.; Tonin, L.; Saeedi, S.; Schneider, C.; Millán, J. del R. The Cybathlon BCI race: Successful longitudinal mutual learning with two tetraplegic users. PLoS Biol. 2018, 16, 1-28.

73. Nuyujukian, P.; Albites Sanabria, J.; Saab, J.; Pandarinath, C.; Jarosiewicz, B.; Blabe, C.H.; Franco, B.; Mernoff, S.T.; Eskandar, E.N.; Simeral, J.D.; et al. Cortical control of a tablet computer by people with paralysis. PLoS One 2018, 13, e0204566.

74. Yu, T.; Li, Y.; Long, J.; Gu, Z. Surfing the internet with a BCI mouse. J. Neural Eng. 2012, 9.

75. Karim, A.A.; Hinterberger, T.; Richter, J.; Mellinger, J.; Neumann, N.; Flor, H.; Kübler, A.; Birbaumer, N. Neural Internet: Web surfing with brain potentials for the completely paralyzed. Neurorehabil. Neural Repair 2006, 20, 508-515.

76. Pennington, C.; McCoy, K.F.; Trnka, K.; McCaw, J.; Yarrington, D. The effects of word prediction on communication rate for AAC. In Proceedings of the Proceedings of NAACL HLT 2007; Rochester, NY, 2007; pp. 173-176.

77. Alomari, M.H.; Abubaker, A.; Turani, A.; Baniyounes, A.M.; Manasreh, A. EEG Mouse : A Machine Learning-Based Brain Computer Interface. Int. J. Adv. Comput. Sci. Appl. 2014, 5, 193-198.

78. Higginbotham, D.J.; Lesher, G.W.; Moulton, B.J.; Roark, B. The application of natural language processing to augmentative and alternative communication. Assist. Technol. 2012, 24, 14-24.

79. Trnka, K.; Yarrington, D.; McCoy, K.; Pennington, C. Topic modeling in fringe word prediction for AAC. IUI 2006, 276-282.

80. Müller, K.R.; Krauledat, M.; Dornhege, G.; Curio, G.; Blankertz, B. Machine Learning and Applications for Brain-Computer Interfacing. In Human Interface and the Management of Information. Methods, Techniques and Tools in Information Design.; Springer: Berlin, Heidelberg, 2007; Vol. 4557, p. 132.

81. Shenoy, P.; Krauledat, M.; Blankertz, B.; Rao, R.P.N.; Müller, K.R. Towards adaptive classification for BCI. J. Neural Eng. 2006, 3.

82. Alamsaputra, D.M.; Kohnert, K.J.; Munson, B.; Reichle, J. Synthesized speech intelligibility among native speakers and non-native speakers of English. Augment. Altern. Commun. 2006, 22, 258-268.

83. Beukelman, D.R.; Mirenda, P. Augmentative and Alternative Communication: Supporting Children and Adults with Complex Communication Needs; 4th ed.; Paul H. Brookes Pub.: Baltimore, 2013.

84. Zhang, X.; Kulkarni, H.; Morris, M.R. Smartphone-Based Gaze Gesture Communication for People with Motor Disabilities. In Proceedings of the Proceedings of the $2017 \mathrm{CHI}$ Conference on Human Factors in Computing Systems; Colorado, USA, 2017; pp. 2878-2889.

85. Villanueva, A.; Cabeza, R.; Porta, S. Eye tracking system model with easy calibration. In Proceedings of the Proceedings of the 2004 symposium on Eye tracking research \& applications; San Antonio, Texas, 2004 ; Vol. 1, p. 55.

86. Sellers, E.W.; Vaughan, T.M.; Wolpaw, J.R. A brain-computer interface for long-term independent home use. Amyotroph. Lateral Scler. 2010, 11, 449-455.

87. Brumberg, J.S.; Pitt, K.M.; Mantie-Kozlowski, A.; Burnison, J.D. Brain-computer interfaces for augmentative and alternative communication: A tutorial. Am. J. Speech-Language Pathol. 2018, 27, 1-12.

88. Abdulkader, S.N.; Atia, A.; Mostafa, M.-S.M. Brain computer interfacing: Applications and challenges. Egypt. Informatics J. 2015, 16, 213-230.

89. Kumar, M. Reducing the Cost of Eye Tracking Systems. Citeseer 2008, 4.

90. Courtney, V.E.; Koverb, S.T. An Open Conversation on Using Eye-Gaze Methods in Studies of Neurodevelopmental Disorders. J. Speech, Lang. Hear. Res. 2015 58, 1719-1732.

91. Kok, E.M.; Jarodzka, H. Before your very eyes: The value and limitations of eye tracking in medical education. Med. Educ. 2017, 51, 114-122. 
92. Wang, Y. Te; Wang, Y.; Cheng, C.K.; Jung, T.P. Developing stimulus presentation on mobile devices for a truly portable SSVEP-based BCI. In Proceedings of the Proceedings of the Annual International Conference of the IEEE Engineering in Medicine and Biology Society, EMBS; IEEE: Osaka, Japan, 2013; pp. 5271-5274.

93. Waller, A. Telling tales: unlocking the potential of AAC technologies. Int. J. Lang. Commun. Disord. 2019, $1-11$.

94. Tauroza, S.; Allison, D. Speech rates in British English. Appl. Linguist. 1990, 11, 90-105.

95. Wilkinson, K.M.; Mitchell, T. Eye Tracking Research to Answer Questions about Augmentative and Alternative Communication Assessment and Intervention. Augment Altern Commun 2015, 30, 106-119.

96. Costigan, F.A.; Newell, K.M. An analysis of constraints on access to augmentative communication in cerebral palsy. Can. J. Occup. Ther. 2009, 153-161.

97. Kumar, S.; Aishwaraya, B.K.; Bhanutheja, K.N.; M, C. Breath to speech communication with fall detection for Elder/Patient with take care analytics. 2016 IEEE Int. Conf. Recent Trends Electron. Inf. Commun. Technol. (RTEICT) Proc; Bangalore, India, 2016, 527-531.

98. Moore, M.M. Real-World Applications for Brain - Computer Interface Technology. IEEE Trans. Neural Syst. Rehabil. Eng. 2003, 11, 162-165.

99. Ruan, S.; Wobbrock, J.O.; Liou, K.; Ng, A.; Landay, J. Speech is $3 x$ faster than typing for english and mandarin text entry on mobile devices. arXiv Prepr. arXiv1608.07323 2016.

100. Baxter, S.; Enderby, P.; Evans, P.; Judge, S. Interventions using high-technology communication devices: A state of the art review. Folia Phoniatr. Logop. 2012, 64, 137-144. 